\title{
Errata: Hardware implementation algorithm and error analysis of high-speed fluorescence lifetime sensing systems using center-of-mass method
}

\section{Day-Uei Li}

Bruce Rae

University of Edinburgh

Institute for Integrated Micro and Nano Systems (IMNS)

School of Engineering

Faraday Building, The King's Buildings

Edinburgh EH9 3JL, Scotland

\section{Robin Andrews}

University of Edinburgh

School of Chemistry

Joseph Black Building, Kings Buildings

Edinburgh EH9 3JJ, Scotland

\section{Jochen Arlt}

University of Edinburgh

School of Physics and Astronomy

Joseph Black Building, The King's Buildings

Edinburgh EH9 3JJ, Scotland

\section{Robert Henderson}

University of Edinburgh

Institute for Integrated Micro and Nano Systems (IMNS)

School of Engineering

Faraday Building, The King's Buildings

Edinburgh EH9 3JL, Scotland

[DOI: $10.1117 / 1.3428872]$

This article [J. Biomed. Opt. 15, 017006 (2010)] was originally published online on 16 February 2010 with errors. The following corrections were made on 23 February 2010:

1) In the affiliation for Robert Henderson appearing on page 1, the "Institute for Micro and Nano System" was changed to "Institute for Integrated Micro and Nano Systems (IMNS)" and "School of Engineering and Electronics" was changed to "School of Engineering."

2) In the corresponding author footnote appearing on page 1, "School of Engineering and Electronics" was changed to "School of Engineering."
3) In Ref. 24 appearing on page 10, "Xlinx, Inc." was changed to "Xilinx, Inc."

In addition, the following correction was made on 2 March 2010: the definition appearing in the second column of page 3 below Eq. (4) appeared as "where $x=\exp (-t / \tau)$ " and was changed to "where $x=\exp (-h / \tau)$."

All versions of the article have been corrected and the article appears correctly in print. 